\title{
De la maîtrise de la langue aux pratiques langagières des élèves : langage et citoyenneté
}

\section{Elisabeth Bautier}

\section{(2) OpenEdition \\ 1 Journals}

\section{Édition électronique}

URL : http://journals.openedition.org/trema/1697

DOI : 10.4000/trema.1697

ISSN : 2107-0997

\section{Éditeur}

Faculté d'Éducation de l'université de Montpellier

\section{Édition imprimée}

Date de publication : 1 octobre 1999

Pagination : 51-57

ISSN : 1167-315X

\section{Référence électronique}

Elisabeth Bautier, « De la maîtrise de la langue aux pratiques langagières des élèves : langage et

citoyenneté », Tréma [En ligne], 15-16 | 1999, mis en ligne le 01 octobre 1999, consulté le 19 avril 2019. URL : http://journals.openedition.org/trema/1697 ; DOI : 10.4000/trema.1697

Ce document a été généré automatiquement le 19 avril 2019.

Trema 


\title{
De la maîtrise de la langue aux pratiques langagières des élèves: langage et citoyenneté $e^{1}$
}

\author{
Elisabeth Bautier
}

1 Traiter de façon conjointe la question des savoirs, du langage et de la citoyenneté, comme tente de le faire ce colloque, permet de penser le langage en ce qu'il est partie prenante de l'exercice de la citoyenneté. Il intervient à plusieurs titres qu'il est important de distinguer, au moins dans un premier moment.

2 Le langage contribue à l'exercice de la citoyenneté en ce qu'il est moyen de construire et de porter une parole, d'exister simultanément en tant que sujet singulier et en tant que membre des circuits de communication, ce qui permet non seulement d'exprimer des idées mais aussi de participer à leur élaboration. A côté de ce domaine de la communication et de l'expression, on rencontre un deuxième registre sur lequel le langage est partie prenante de la citoyenneté, c'est celui de son rôle dans la construction, l'élaboration, l'appropriation des savoirs et ce faisant, dans le partage d'une culture commune et de références qui fonde l'appartenance à la communauté des citoyens.

3 Le domaine de la communication et de l'expression est celui qui fait l'objet explicite des apprentissages scolaires: des enseignements sont censés apprendre aux élèves à communiquer, à mieux s'exprimer. Cette centration sur l'expression et la communication déconnectée des usages du langage pour apprendre, se saisir du monde et l'élaborer, penser, construire un point de vue, non seulement ne pose pas toujours la question de la construction sociale et cognitive des objets mêmes de la communication et de l'expression, mais correspond pour une grande part à la dissociation fréquente entre socialisation et apprentissage et à la valorisation de la première; c'est du moins le cas pour certains élèves considérés comme moins "socialisés » que d'autres, comme si la socialisation pouvait se construire indépendamment des références communes, d'univers de pensée partagés, qui permettent non pas le consensus et l'uniformité mais justement le débat, la confrontation, l'espace commun d'échanges d'idées. Tout se passe comme si 
certains élèves relevaient plus que d'autres de l'expression de soi et de la communication sociale, tandis que d'autres seraient dans une logique de connaissance et de construction de la pluralité des points de vue, celle qui permet l'analyse et le commentaire des textes et du monde.

Penser les différentes composantes de l'activité langagière et les différents usages du langage comme indépendants les uns des autres conduit à penser ces derniers comme relevant de savoirs enseignables, de techniques, voire d'actes langagiers plus que comme une activité sociale et toujours simultanément cognitive, subjective, référentielle. Le langage est pratique et activité avant d'être savoirs et compétences et c'est ce qu'une perspective de formation d'un citoyen ne peut laisser de côté au risque de confondre restitution de savoirs linguistiques et élaboration langagière. L'activité langagière n'est pas réductible à des savoirs enseignés que l'on mobilise plus ou moins consciemment, elle est aussi expérience du monde et des autres, valeurs, habitudes de vie, elle est un lieu privilégié de l'élaboration, de la transformation de soi. S'il ne s'agit pas de rejeter l'intérêt des acquisitions «techniques ", qui peuvent être des moments dans des transformations plus profondes et plus larges, et qui sont pour une part au moins nécessaires, il s'agit cependant de rappeler que ces compétences, compétence communicatives, textuelles, linguistiques, n'épuisent pas les objets d'apprentissages langagiers, qu'elles ne changent que très rarement le rapport des élèves au langage, au savoir et à l'apprentissage. ${ }^{2}$

\section{De la nécessité pour l'école de se saisir de la dimension plurielle du langage alors même que l'attention est souvent portée sur la maîtrise de la langue}

\section{Langage et savoirs : des usages langagiers différenciateurs}

5 Des constats et une ambition nécessaire pour des élèves pensés comme citoyens conduisent donc à remettre en question une approche de la question linguistique centrée sur la seule maîtrise de la langue et de ses formes écrites telle que les enseignants et enseignements de "français » ont l'habitude de la décrire et de l'évaluer. Il y a au demeurant une grande difficulté à ne pas réduire les problèmes de langage et d'apprentissage à des problèmes de lecture, de syntaxe fautive ou de vocabulaire "pauvre", en d'autres termes, il est difficile de penser ensemble les formes de la langue et les usages du langage oraux et écrits et ce qu'ils permettent de faire au regard des exigences des activités scolaires d'apprentissage. Des problèmes identifiés comme relevant de la langue ne sont que rarement «seulement » des problèmes de langue : ils correspondent le plus souvent à des questions d'usages du langage. Privilégier la maitrise de la langue peut conduire à un oubli peut-être majeur dans les démarches d'enseignement, celui du langage, de l'écrit en particulier, pour apprendre, élaborer, alors même que cet usage du langage est justement fortement différenciateur des élèves, que comme pour l'ensemble des pratiques langagières, ces utilisations du langage relèvent d'une familiarisation avec des pratiques, un rapport au langage, au savoir, des valeurs qui ne sont pas partagés par tous. Se trouve ainsi posée la question de la réalité de la démocratisation d'un enseignement qui ignore ce domaine de l'activité langagière. 
6 En effet, parce que l'enseignement de la langue, de l'expression et de la communication ne suffisent pas pour transformer des pratiques, parce qu'il peut y avoir prégnance chez certains élèves d'un rapport au langage et de pratiques langagières qui transcendent les situations qu'elles soient d'oralité ou d'écriture, on retrouve des constantes dans les manières de faire avec le langage, des postures, des points de vue, privilégiés quand il s'agit de construire des savoirs (pas seulement de les restituer) tout au moins dans les situations scolaires ; ces postures et manières de faire ne sont pas équivalentes quant il s'agit de s'approprier des savoirs simultanément langagiers et disciplinaires. Par exemple, parce que la pratique du commentaire de textes, voire de la dissertation, comme les autres activités langagières, n'est pas réductible à un ensemble de techniques et d'outils, et malgré un enseignement de ces types d'écrit, on retrouve des réalisations de ces écrits scolaires qui correspondent à des manières de faire avec le langage relativement récurrentes pour chacun. Selon les élèves, le commentaire sera simplement redite paraphrasée du texte, de la consigne, ou l'occasion de manifester son opinion, son affectivité, son émotion, ses valeurs personnelles, ou encore des connaissances qui font sortir du texte et du thème ; il sera, pour les élèves qui confondent l'activité langagière et la tâche scolaire, elle-même pensée comme simple restitution de savoirs scolaires, l'utilisation systématique des outils de commentaire enseignés, ou d'un schéma textuel qui fonctionne "avide». Un commentaire, une dissertation, un écrit d'histoire ou de biologie, lorsqu'ils ne se réduisent pas à l'effectuation de l'exercice scolaire, sont le fait des élèves qui sont auteurs de leurs écrits qu'ils élaborent en élaborant des connaissances simultanément langagières, linguistiques et disciplinaires dans le cadre des genres scolaires spécifiques de la discipline, à condition qu'il en ait appris le sens « intellectuel » et pas seulement les règles de réalisation formelle ${ }^{3}$.

Paysannes péruviennes apprenant l'alphabet. Photo d'une exposition itinérante organisée par I'UNESCO pour l'Année Internationale de l'Alphabétisation.

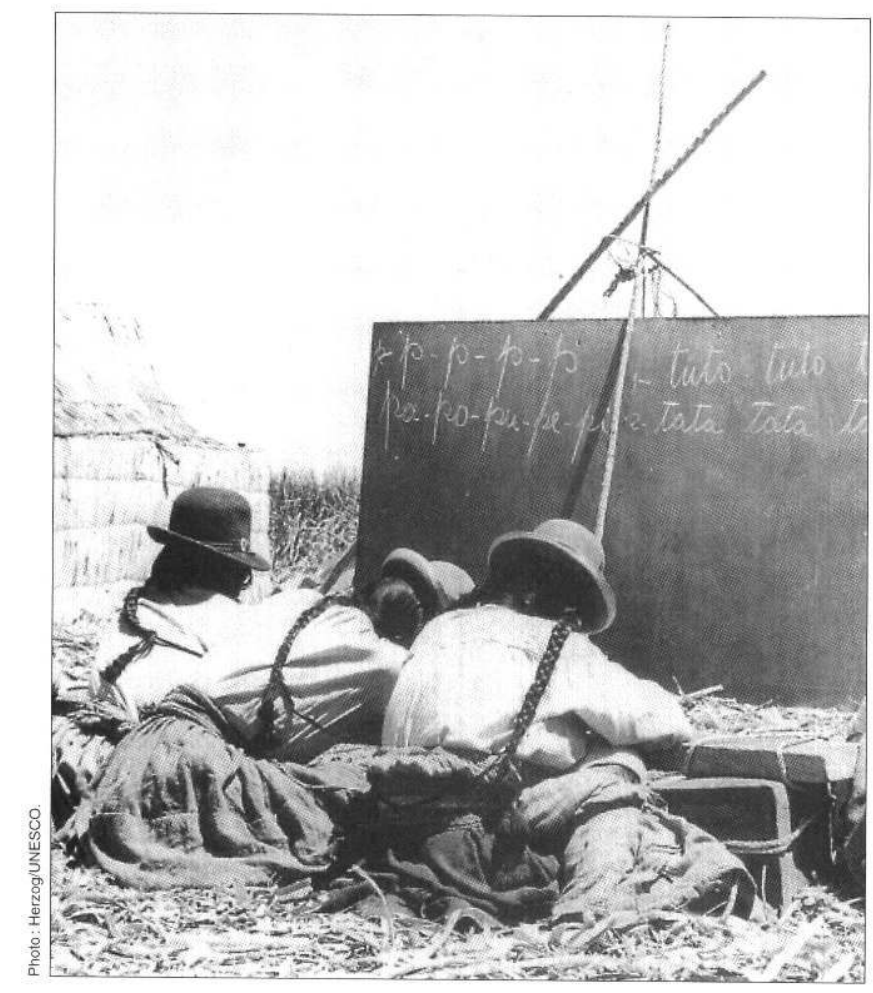


Le Courrier de I'UNESCO. Paris, UNESCO, Juillet 1990 (Vol. 43, N7), 50 p., p. 26.

7 En effet, l'école, malgré le discours sur la maitrise de la langue, a en fait des exigences d'une toute autre nature : elle se situe dans une familiarité supposée - alors même que celle-ci est rare - avec l'utilisation du langage pour élaborer, y compris des connaissances à partir de l'expérience, elle valorise les pratiques langagières d'oralité et d'écriture qui ont à voir avec le travail intellectuel, alors même que leur apprentissage n'est que rarement explicite. Dans la grande majorité des cas, est explicitée la tâche scolaire dans sa réalisation quasi-matérielle, mais cette explicitation laisse de côté la face cognitive, ou plutôt socio-cognitive de l'activité, liée aux formes scolaires, disciplinaires même socialement et historiquement construites : ainsi décrire en français, en mathématiques, en biologie, en physique et en histoire ne renvoie pas aux mêmes activités sociocognitivo-langagières. Pourtant, se taire sur ce qu'il y a à faire conjointement avec la langue, le langage et les savoirs est producteur d'inégalité et ce n'est pas seulement une question de langue : savoir que décrire le cycle de vie du cerisier en biologie ne suppose pas que l'on mentionne un jardinier et ou que l'on aime les cerises ne relève pas de la maitrise de la langue, mais d'un ensemble de savoirs hétérogènes concernant ce que l'on peut faire avec le langage, ce qu'est un savoir en biologie, ce qu'est écrire un savoir dans le genre scolaire de la biologie.

Un autre exemple de pratique langagière "gênante " pour s'approprier et construire des savoirs est donné par les élèves (et des étudiants) qui ont du mal à travailler la langue, c'est-à-dire à avoir avec le langage des pratiques d'élaboration, de réécriture, de retour sur le déjà écrit, ils sont le plus souvent dans l'écriture au fil de la plume, comme dans le flux de la parole et au-delà dans l'adhérence à ce qu'ils disent et ce qu'ils font, dans la transparence de la parole comme du monde. Ce sont aussi ceux, il y a cohérence, qui confondent savoirs et vérité du monde quasi intemporelle, qui ne « reconnaissent » pas la notion de théorie puisque le monde est vécu sur le mode de l'évidence. Ces exemples suggèrent que rapport au langage et rapport au savoir peuvent difficilement être dissociés, de là l'importance du premier dans le processus de démocratisation, de construction de savoirs partagés, que penser la question de la langue en termes de compétences minimales ou de base est beaucoup trop réducteur pour être pertinent dans une perspective de formation des élèves. Ce qui ne veut pas dire que la connaissance de la syntaxe, de la morphologie ou de la syntaxe de la phrase ne sont pas des savoirs nécessaires, seulement qu'ils ne constituent pas un minimum ou une base.

\section{Certaines pratiques d'enseignement plus que d'autres aident les élèves}

9 A mobilisation initiale équivalente des élèves, les malentendus portant sur les postures langagières et les activités intellectuelles requises par l'appropriation des savoirs peuvent, lorsque le fonctionnement de l'institution scolaire et les pratiques de ses professionnels ne permettent pas de les lever, a fortiori lorsqu'ils contribuent à les créer ou à les renforcer, leurrer certains élèves quant à la nature du travail et des activités cognitives et langagières pertinentes pour apprendre et, par là, les conduire à des situations d'échec d'autant plus mal vécues qu'ils n'auront pu en comprendre les causes. 
10 Dès lors, des modes de travail visant à subjectiver, finaliser et contextualiser les apprentissages et qui vont bien souvent de pair avec le souci d'accorder une grande importance à la référence au " vécu ", à l'expression de l'expérience de l'élève, ou plutôt de l'enfant ou de l'adolescent font courir à certains élèves le risque du malentendu, à ceux-là même pour qui ces usages du langage sont les plus familiers, voire sont ceux qu'ils privilégient. Pour ces élèves, la constitution de cette expérience en objet de réflexion et de pensée, plus encore son élaboration en savoir ne vont pas de soi, pas plus que la nécessaire distinction, tout à la fois cognitive et langagière, entre ce que l'on pourrait appeler le «moi-je» de l'expérience vécue et du rapport pratique aux situations immédiates, et le " je » objectivé d'un rapport réflexif à cette expérience, au monde et à soi-même. Lorsque ces processus ont déjà été construits chez l'élève, le recours et la référence à l'expérience personnelle et à l'expression de soi dans le cadre scolaire peuvent certes donner lieu pour lui à un travail d'élaboration de l'expérience en savoir. Mais, ce n'est pas le cas pour tous. Les construire est alors tâche nécessaire de l'enseignement. $^{4}$

11 J. Bernardin en souligne certaines conditions ${ }^{5}$ ? D'abord permettre à l'élève de prendre la parole; puis, construire des situations d'exploration ouvertes y est propice, mais trop simples, elles manquent d'enjeu stimulant ; trop fermées, elles ne favorisent que ceux qui anticipent les attendus scolaires et fonctionnent par «clin d'œil » avec l'enseignant, trop convergentes, elles sont peu propices à la diversité ; enfin, organiser la confrontation à l'exigence : solliciter et accueillir la parole des élèves, ce n'est pas négligeable... mais il y a risque de glisser dans un relativisme culturel tout aussi discriminant qu'une confrontation «sèche » à la normalisation langagière qui stigmatise celui qui "parle mal »... Pour certains élèves qui parlent en multipliant les ellipses, se satisfont de l'à-peuprès au vu du contexte, voire qui complètent leurs propos par le geste et considèrent les exigences scolaires superflues parce que confondues avec un arbitraire, il est nécessaire de faire ressentir le besoin de préciser, de devenir plus exigeant (employer le terme correct). Il s'agit donc que les normes toujours alors simultanément linguistiques, langagières, communicatives, s'imposent d'elles-mêmes non qu'elles paraissent s'imposer dans un arbitraire institutionnel et social vécu alors sur le registre de l'incompréhension réciproque et de la violence symbolique.

On pourra prendre appui sur l'expérience commune afin de constituer un référent identique, mais aussi de faire fond sur un rapport pragmatique au monde qu'on sait être plus caractéristique des élèves qui nous préoccupent. Faire d'abord (classer, construire, dessiner...) pour solliciter les points de vue, tous les points de vue... Le débat qui suit permet le développement des explications, des hypothèses, l'échange des points de vue. C'est l'occasion d'une mise à distance des premières manières de voir, toutes empreintes de subjectivité. Pour que le conflit de points de vue soit fructueux et amène à une réelle élaboration collective, il faut s'empêcher de favoriser trop hâtivement la «bonne réponse ", voire provoquer de la turbulence dans le ronronnement de la pensée : rappeler les avis opposés, exacerber les contradictions et les ambiguïtés pour sortir de l'implicite, pousser à davantage de précision. Ne pas donner la solution donc, mais plutôt renvoyer en miroir grossissant les éléments qui pousseront à faire de nouvelles mises en relation, à inventer des solutions originales. Relatif "effacement» du maître donc pour que la parole se construise chez les élèves : ni parole défouloir, ni bavardage sympathique, ni simple expression de son avis, mais parole qui engage et doit trouver un étayage face aux oppositions, s'imposer par la pertinence de son argumentation face au problème à 
résoudre. Ce n'est pas l'enseignant, mais le réel (à comprendre) qui sanctionne... Le choc avec la pensée des autres pousse chacun à la prise de conscience des faiblesses des propositions singulières et de la nécessité d'une " codification » commune.

13 A trop focaliser sur la maitrise de la langue, les enseignants se heurtent à une impasse. Travailler simultanément trois dimensions du langage: construire avec les élèves la langue comme pratique (moyen de dire et moyen de faire), comme système linguistique, et comme manière de penser et de construire un univers de référence est une exigence majeure pour que l'élève occupe une place sociale pleine et entière dans le monde social.

\section{NOTES}

1. Une partie de ce texte a fait l'objet d'une communication au $7^{\mathrm{e}}$ colloque de l'association internationale de didactique du français langue maternelle qui s'est tenu en septembre 1998 à Bruxelles sur le thème Quels savoirs pour quelles valeurs?

2. Cf., pour une approche critique générale de ce type de logique - ROPÉ F. et TANGUY L. (dir.) : Savoirs et compétences. De l'usage de ces notions dans l'école et l'entreprise. Paris, L'Harmattan, 1994.

3. Cette question est développée en collaboration avec D. Bucheton, dans l'ouvrage - BAUTIER É. et ROCHEX J.-Y. : L'expérience scolaire des nouveaux lycéens. Démocratisation ou massification? Paris, A. Colin, 1998.

4. Ce thème est développé dans - BAUTIER É. et ROCHEX J.-Y. : «Ces malentendus qui font la différence ", in La scolarisation de la France, (sous la dir. de : TERRAIL J.-P). Paris, La Dispute, 1997.

5. Communication aux assises nationales de la relance des ZEP, juin 1998. Ces démarches font l'objet de l'ouvrage Comment les enfants entrent dans la culture écrite, Paris, Retz, 1997. A ces mêmes assises, Alain Decron, professeur des écoles à Perpignan, a donné d'autres exemples de telles démarches. Il travaille dans une classe de CE1 composée pour $70 \%$ d'élèves d'origine berbère et possédant donc une tradition orale. En géographie, les enfants ont visionné en début d'année une cassette sur les Inuits avec la consigne de prendre des notes pendant une heure sur un cahier de travail. Ces écrits, non élaborés donc, ont ensuite été lus et discutés en classe. Le but était de faire comprendre aux élèves que l'écriture est un moyen de garder une trace mais surtout un moyen de travailler ce que l'on est en train d'apprendre, de penser ensemble puis de repenser seul. Cette dimension de l'écriture n'est pas familière à tous les enfants, c'est donc le rôle de l'école de leur apporter cette dimension. L'écrit va ensuite permettre aux élèves de faire le parcours de leur propre point de vue : en partant de leurs notes, ils vont débattre et ce débat va nourrir leur pensée. La démarche est ensuite de faire des va-et-vient incessants entre l'écrit des élèves et l'oral afin de structurer la pensée et les savoirs et d'expliquer ce qu'ils ont vu appris. Une série de réécritures suit le premier débat, elles introduisent une norme d'écriture plus scolaire que celle des prises de notes. Les enfants apprennent à se servir du langage pour médiatiser leur savoir. Notons qu'ils argumentent non pas pour convaincre, ce qu'ils savent faire, mais pour penser ensemble et pour chacun. 


\section{RÉSUMÉS}

L'approche linguistique centrée sur la seule maîtrise de la langue est remise en question. Il faudrait doter les élèves d'une maîtrise du langage permettant à tous de construire les apprentissages scolaires. Est posée la question de la réalité de la démocratisation d'un enseignement qui ignore ce domaine de l'activité langagière.

This article questions the linguistic approach to the French language of focusing solely upon the mastery of language usage. The author asserts that students should be given instruction in such a language mastery, as may allow one and all of them to acquire the learning processes of the various school disciplines. The issue is considered whether or not an education ignoring this field of language activity is truly democratic.

\section{INDEX}

Keywords : relationship to an intellectual object, social dimension

Mots-clés : dimension sociale, rapport à un objet de savoir

\section{AUTEUR}

\section{ELISABETH BAUTIER}

Université de Paris VIII, équipe ESCOL 